\title{
Handling Non Left-Linear Rules When Completing Tree Automata*
}

\author{
YOHAN BOICHUT \\ LIFO Bat. 3IA Université d'Orléans Rue Léonard de Vinci B.P. 6759 F-45067 ORLEANS \\ ROMEO COURBIS \\ INRIA-CASSIS LIFC, Université de Franche-Comté, 16 route de Gray, 25000 Besançon, \\ FRANCE \\ PIERRE-CYRILLE HEAM \\ LSV, CNRS-INRIA, Ecole Normale Supérieure de Cachan, 61 avenue du Président Wilson, \\ 94230 Cachan, FRANCE \\ OLGA KOUCHNARENKO \\ INRIA-CASSIS LIFC, Université de Franche-Comté, 16 route de Gray, 25000 Besançon, \\ FRANCE
}

\author{
Received (Day Month Year) \\ Accepted (Day Month Year) \\ Communicated by (xxxxxxxxxx)
}

\begin{abstract}
This paper addresses the following general problem of tree regular model-checking: decide whether $\mathcal{R}^{*}(\mathcal{L}) \cap \mathcal{L}_{p}=\emptyset$ where $\mathcal{R}^{*}$ is the reflexive and transitive closure of a successor relation induced by a term rewriting system $\mathcal{R}$, and $\mathcal{L}$ and $\mathcal{L}_{p}$ are both regular tree languages. We develop an automatic approximation-based technique to handle this undecidable in general - problem in the case when term rewriting system rules are non left-linear.
\end{abstract}

Keywords: Rewriting techniques; tree automata; left-linearity; security.

\section{Introduction}

Automatic verification of software systems is one of the most challenging research problems in computer aided verification. In this context, regular model-checking has been proposed as a general framework for analysing and verifying infinite state systems. In this framework, systems are modelled using regular representations: the systems configurations are modelled by finite words or trees (of unbounded size) and the dynamic behaviour of systems is modelled either by a transducer or a (term) rewriting system (TRS for short). Afterwards, a system reachability-based analysis

*This work was granted by the French ANR project RAVAJ. 
is reduced to the regular languages closure computation under (term) rewriting systems: given a regular language $\mathcal{L}$, a relation $\mathcal{R}$ induced by a (term) rewriting system and a regular set $\mathcal{L}_{P}$ of bad configurations, the problem is to decide whether $\mathcal{R}^{*}(\mathcal{L}) \cap \mathcal{L}_{p}=\emptyset$ where $\mathcal{R}^{*}$ is the reflexive and transitive closure of $\mathcal{R}$. Since $\mathcal{R}^{*}(\mathcal{L})$ is in general neither regular nor decidable, several approaches handle restricted cases of this problem.

In this paper we address this problem for tree regular languages by automatically computing over- and under-approximations of $\mathcal{R}^{*}(\mathcal{L})$. Computing an overapproximation $K_{\text {over }}$ of $\mathcal{R}^{*}(\mathcal{L})$ may be useful for the verification if $K_{\text {over }} \cap \mathcal{L}_{p}=\emptyset$, proving that $\mathcal{R}^{*}(\mathcal{L}) \cap \mathcal{L}_{p}=\emptyset$. Dually, under-approximation may be suitable to prove that $\mathcal{R}^{*}(\mathcal{L}) \cap \mathcal{L}_{p} \neq \emptyset$ (see Fig.1). This approach is relevant if the computed approximations are not too coarse. Another important point is that in general, there are some restrictions on the rewriting systems in order to ensure the soundness of the above approach. This paper follows and adapts an expert-human guided approximation technique introduced in [17] for left-linear term-rewriting systems. More precisely, the paper 1) extends this approach to term rewriting systems with nonleft-linear rules, 2) illustrates its advantages on examples, and 3) points out the application domains.

Notice that a preliminary version of the present paper has been published [7]. The results in [7] are obtained for left-quadratic TRSs. To make it short, the present paper extends the model in [7] and generalises the underpinning constructions to deal with all non left-linear term rewriting systems. In addition, more examples are given to illustrate the definitions.

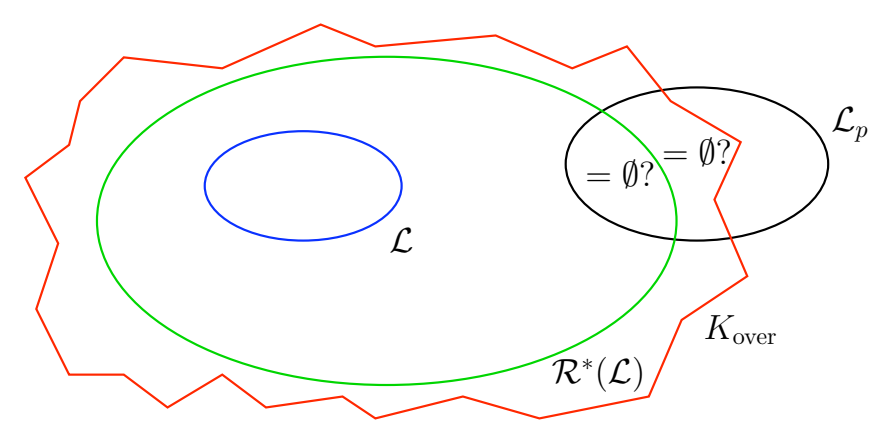

Figure 1 . Is the intersection between $K_{\text {over }}$ and $\mathcal{L}_{p}$ empty?

Related Work Given a term rewriting system $\mathcal{R}$ and two ground terms $s$ and $t$, deciding whether $s \rightarrow_{\mathcal{R}}^{*} t$ is a central question in automatic proof theory. This problem is shown decidable for term rewriting systems which are terminating but it is undecidable in general. Several syntactic classes of term rewriting systems have been pointed out to have a decidable accessibility problem, for in- 
stance by providing an algorithm to compute $\mathcal{R}^{*}(\mathcal{L})$ when $\mathcal{L}$ is a regular tree language $[15,13,19,22,25,26,23]$. In [17], the authors focus on a general completion based human-guided technique. This technique has been successfully used (not automatically) to prove the security of cryptographic protocols [18] and recently Java Bytecode programs [4]. This framework was extended in [24] to languages accepted by AC-tree automata. We quote several works for tree regular model checking proposed in $[9,1,8,20]$.

Layout of the paper The paper is organised as follows. Section 2 introduces notations and the basic completion approach. Next, Section 3 presents the main theoretical contributions of the paper, while Section 4 describes a family of examples and gives related security issues. Finally, Section 5 concludes.

\section{Preliminaries}

\subsection{Terms and TRSs}

Comprehensive surveys can be found in $[16,2]$ for term rewriting systems, and in $[12,19]$ for tree automata and tree language theory.

Let $\mathcal{F}$ be a finite set of symbols, associated with an arity function ar $: \mathcal{F} \rightarrow \mathbb{N}$, and let $\mathcal{X}$ be a countable set of variables. $\mathcal{T}(\mathcal{F}, \mathcal{X})$ denotes the set of terms, and $\mathcal{T}(\mathcal{F})$ denotes the set of ground terms (terms without variables). The set of variables of a term $t$ is denoted by $\operatorname{Var}(t)$. A substitution is a function $\sigma$ from $\mathcal{X}$ into $\mathcal{T}(\mathcal{F}, \mathcal{X})$, which can be extended uniquely to an endomorphism of $\mathcal{T}(\mathcal{F}, \mathcal{X})$. A position $p$ for a term $t$ is a word over $\mathbb{N}$. The empty sequence $\epsilon$ denotes the top-most position. The set $\operatorname{Pos}(t)$ of positions of a term $t$ is inductively defined by: $\operatorname{Pos}(t)=\{\epsilon\}$ if $t \in \mathcal{X}$ and $\mathcal{P} o s\left(f\left(t_{1}, \ldots, t_{n}\right)\right)=\{\epsilon\} \cup\left\{i . p \mid 1 \leq i \leq n\right.$ and $\left.p \in \mathcal{P} o s\left(t_{i}\right)\right\}$. If $p \in \mathcal{P}$ os $(t)$, then $\left.t\right|_{p}$ denotes the subterm of $t$ at position $p$ and $t[s]_{p}$ denotes the term obtained by replacement of the subterm $\left.t\right|_{p}$ at position $p$ by the term $s$. We also denote by $t(p)$ the symbol occurring in $t$ at position $p$. Given a term $t \in \mathcal{T}(\mathcal{F}, \mathcal{X})$, we denote $\mathcal{P}_{0} s_{A}(t)$ $(\subseteq \mathcal{P}$ os $(t))$ the set of positions of $t$ such that $\mathcal{P}_{o s}(t)=\{p \in \mathcal{P} o s(t) \mid t(p) \in A\}$. Thus $\operatorname{Pos}_{\mathcal{F}}(t)$ is the set of functional positions of $t$.

A term rewriting system (TRS) $\mathcal{R}$ is a set of rewrite rules $l \rightarrow r$, where $l, r \in$ $\mathcal{T}(\mathcal{F}, \mathcal{X})$ and $l \notin \mathcal{X}$. A rewrite rule $l \rightarrow r$ is $h$-left-linear (resp. $h$-right-linear) if each variable of $l$ (resp. $r$ ) occurs at most $h$ times within $l$ (resp. $r$ ). A TRS $\mathcal{R}$ is $h$-leftlinear (resp. $h$-right-linear) if every rewrite rule $l \rightarrow r$ of $\mathcal{R}$ is $h$-left-linear (resp. $h$-right-linear). For above linearity definitions, if $h=1$, the prefix " $h-$ " is omitted. A TRS $\mathcal{R}$ is linear if it is right-linear and left-linear. The TRS $\mathcal{R}$ induces a rewriting relation $\rightarrow_{\mathcal{R}}$ on terms whose reflexive transitive closure is written $\rightarrow_{\mathcal{R}}^{*}$. The set of $\mathcal{R}$ descendants of a set of ground terms $E$ is $\mathcal{R}^{*}(E)=\left\{t \in \mathcal{T}(\mathcal{F}) \mid \exists s \in E\right.$ s.t. $\left.s \rightarrow_{\mathcal{R}}^{*} t\right\}$. For every positive integer $k$, any ground terms $s$ and $t$, we inductively define $s \rightarrow{ }_{\mathcal{R}}^{k} t$ by: $s \rightarrow_{\mathcal{R}}^{0} t$ if and only if $s=t$, and $s \rightarrow_{\mathcal{R}}^{k+1} t$ if and only if there exists a term $s_{1}$ such that $s_{1} \rightarrow_{\mathcal{R}}^{k} t$ and $s \rightarrow_{\mathcal{R}} s_{1}$. 


\subsection{Tree Automata Completion}

Note that $\mathcal{R}^{*}(E)$ is possibly infinite: $\mathcal{R}$ may not terminate and/or $E$ may be infinite. The set $\mathcal{R}^{*}(E)$ is generally not computable [19]. However, it is possible to over-approximate it [17] using tree automata, i.e. a finite representation of infinite (regular) sets of terms. We next define tree automata.

Let $\mathcal{Q}$ be a finite set of symbols, of arity 0 , called states such that $\mathcal{Q} \cap \mathcal{F}=\emptyset$. $\mathcal{T}(\mathcal{F} \cup \mathcal{Q})$ is called the set of configurations A transition is a rewrite rule $c \rightarrow q$, where $c \in \mathcal{T}(\mathcal{F} \cup \mathcal{Q})$ is a configuration and $q \in \mathcal{Q}$. A normalised transition is a transition $c \rightarrow q$ where $c=f\left(q_{1}, \ldots, q_{n}\right), f \in \mathcal{F}, \operatorname{ar}(f)=n$, and $q_{1}, \ldots, q_{n} \in \mathcal{Q}$. A bottom-up non-deterministic finite tree automaton (tree automaton for short) is a quadruple $\mathcal{A}=\left\langle\mathcal{F}, \mathcal{Q}, \mathcal{Q}_{f}, \Delta\right\rangle, \mathcal{Q}_{f} \subseteq \mathcal{Q}$ and $\Delta$ is a finite set of normalised transitions. The rewriting relation on $\mathcal{T}(\mathcal{F} \cup \mathcal{Q})$ induced by the transition set $\Delta$ of $\mathcal{A}$ is denoted $\rightarrow_{\Delta}$. When $\Delta$ is clear from the context, $\rightarrow_{\Delta}$ is also written $\rightarrow_{\mathcal{A}}$. The tree language recognised by $\mathcal{A}$ in a state $q$ is $\mathcal{L}(\mathcal{A}, q)=\left\{t \in \mathcal{T}(\mathcal{F}) \mid t \rightarrow^{\star}{ }_{\mathcal{A}} q\right\}$. The language recognised by $\mathcal{A}$ is $\mathcal{L}(\mathcal{A})=\bigcup_{q \in \mathcal{Q}_{f}} \mathcal{L}(\mathcal{A}, q)$. A tree language is regular if and only if it is recognised by a tree automaton. We denote by $C\left[q_{1}, \ldots, q_{n}\right]$ a term of $\mathcal{T}\left(\mathcal{F} \cup\left\{q_{1}, \ldots, q_{n}\right\}\right)$.

Let us now recall how tree automata and TRSs can be used for term reachability analysis. Given a tree automaton $\mathcal{A}$ and a TRS $\mathcal{R}$, the tree automata completion algorithm proposed in [17] computes a tree automaton $\mathcal{A}_{\mathcal{R}}^{k}$ such that $\mathcal{L}\left(\mathcal{A}_{\mathcal{R}}^{k}\right)=$ $\mathcal{R}^{*}(\mathcal{L}(\mathcal{A}))$ when it is possible (for the classes of TRSs where an exact computation is possible, see [17]), and such that $\mathcal{L}\left(\mathcal{A}_{\mathcal{R}}^{k}\right) \supseteq \mathcal{R}^{*}(\mathcal{L}(\mathcal{A}))$ otherwise.

The tree automata completion works as follows. From $\mathcal{A}=\mathcal{A}_{\mathcal{R}}^{0}$ completion builds a sequence $\mathcal{A}_{\mathcal{R}}^{0}, \mathcal{A}_{\mathcal{R}}^{1} \ldots \mathcal{A}_{\mathcal{R}}^{k}$ of automata such that if $s \in \mathcal{L}\left(\mathcal{A}_{\mathcal{R}}^{i}\right)$ and $s \rightarrow \mathcal{R} t$ then $t \in \mathcal{L}\left(\mathcal{A}_{\mathcal{R}}^{i+1}\right)$. If there is a fix-point automaton $\mathcal{A}_{\mathcal{R}}^{k}$ such that $\mathcal{R}^{*}\left(\mathcal{L}\left(\mathcal{A}_{\mathcal{R}}^{k}\right)\right)=\mathcal{L}\left(\mathcal{A}_{\mathcal{R}}^{k}\right)$, then $\mathcal{L}\left(\mathcal{A}_{\mathcal{R}}^{k}\right)=\mathcal{R}^{*}\left(\mathcal{L}\left(\mathcal{A}_{\mathcal{R}}^{0}\right)\right)$ (or $\mathcal{L}\left(\mathcal{A}_{\mathcal{R}}^{k}\right) \supseteq \mathcal{R}^{*}(\mathcal{L}(\mathcal{A}))$ if $\mathcal{R}$ is in no class of [17]). To build $\mathcal{A}_{\mathcal{R}}^{i+1}$ from $\mathcal{A}_{\mathcal{R}}^{i}$, a completion step is achieved. It consists of finding critical pairs between $\rightarrow_{\mathcal{R}}$ and $\rightarrow \mathcal{A}_{\mathcal{R}}^{i}$. To define the notion of critical pair, the substitution definition is extended to terms in $\mathcal{T}(\mathcal{F} \cup \mathcal{Q})$. For a substitution $\sigma: \mathcal{X} \mapsto \mathcal{Q}$ and a rule $l \rightarrow r \in \mathcal{R}$ such that $\mathcal{V} a r(r) \subseteq \mathcal{V}$ ar $(l)$, if there exists $q \in \mathcal{Q}$ satisfying $l \sigma \rightarrow_{\mathcal{A}_{\mathcal{R}}^{i}}^{*} q$ then $l \sigma \rightarrow{ }_{\mathcal{A}_{\mathcal{R}}^{i}}^{*} q$ and $l \sigma \rightarrow_{\mathcal{R}} r \sigma$ is a critical pair. Note that since $\mathcal{R}$ and $\mathcal{A}_{\mathcal{R}}^{i}$ is finite, there is only a finite number of critical pairs. Thus, for every critical pair detected between $\mathcal{R}$ and $\mathcal{A}_{\mathcal{R}}^{i}$ such that $r \sigma \nrightarrow^{*}{ }_{\mathcal{A}_{\mathcal{R}}^{i}} q$, the tree automaton $\mathcal{A}_{\mathcal{R}}^{i+1}$ is constructed by adding a new transition $r \sigma \rightarrow q$ to $\mathcal{A}_{\mathcal{R}}^{i}$. Consequently, $\mathcal{A}_{\mathcal{R}}^{i+1}$ recognises $r \sigma$ in $q$, i.e. $r \sigma \rightarrow \mathcal{A}_{\mathcal{R}}^{i+1} q$. However, the transition $r \sigma \rightarrow q$ is not necessarily normalised. Then, we use abstraction functions whose goal is to define a set of normalised transitions Norm such that $r \sigma \rightarrow_{\text {Norm }}^{*} q$. Thus, instead of adding the transition $r \sigma \rightarrow q$ which is not normalised, the set of transitions Norm is added to $\Delta$, i.e., the transition set of the current automaton $\mathcal{A}_{\mathcal{R}}^{i}$.

We give below a very general definition of abstraction functions which allot to each functional position of $r \sigma$ a state of $\mathcal{Q}$. The role of an abstraction function remains to define equivalence classes of terms where one class corresponds to one 
state of $\mathcal{Q}$. An abstraction function $\gamma$ is a function $\gamma:((\mathcal{R} \times(\mathcal{X} \rightarrow \mathcal{Q}) \times \mathcal{Q}) \mapsto$ $\left.\mathbb{N}^{*}\right) \mapsto \mathcal{Q}$ such that $\gamma(l \rightarrow r, \sigma, q)(\epsilon)=q$. Thus, given an abstraction function $\gamma$, the normalisation of a transition $r \sigma \rightarrow q$ is defined as follows. Let $\gamma$ be an abstraction function, $\Delta$ be a transition set, $l \rightarrow r \in R$ with $\mathcal{V} a r(r) \subseteq \mathcal{V} \operatorname{ar}(l)$ and $\sigma: \mathcal{X} \rightarrow \mathcal{Q}$ such that $l \sigma \rightarrow{ }_{\Delta}^{*} q$. The $\gamma-$ normalisation of the transition $r \sigma \rightarrow q$, written $\operatorname{Norm}_{\gamma}(l \rightarrow r, \sigma, q)$, is defined by:

$$
\begin{aligned}
& \operatorname{Norm}_{\gamma}(l \rightarrow r, \sigma, q)=\left\{r(p)\left(\beta_{p .1}, \ldots, \beta_{p . n}\right) \rightarrow \beta \mid\right. \\
& p \in \mathcal{P}_{\mathcal{F}}(r), \\
& \beta=\left\{\begin{array}{l}
q \text { if } p=\epsilon \\
\gamma(l \rightarrow r, \sigma, q)(p) \text { otherwise },
\end{array}\right. \\
& \beta_{p . i}=\left\{\begin{array}{l}
\sigma(r(p . i)) \text { if } r(p . i) \in \mathcal{X} \\
\gamma(l \rightarrow r, \sigma, q)(p . i) \text { otherwise. }
\end{array}\right.
\end{aligned}
$$

Example 1. Let $\mathcal{A}=\left\langle\mathcal{F}, \mathcal{Q}, \mathcal{Q}_{f}, \Delta\right\rangle$ be the tree automaton such that $\mathcal{F}=$ $\{a, b, c, d, e, f, \omega\}$ with $\operatorname{ar}(s)=1$ with $s \in\{a, b, c, d, e, f\}$ and $\operatorname{ar}(\omega)=0, \mathcal{Q}=$ $\left\{q_{b}, q_{f}, q_{\omega}\right\}, \mathcal{Q}_{f}=\left\{q_{f}\right\}$ and $\Delta=\left\{\omega \rightarrow q_{\omega}, b\left(q_{\omega}\right) \rightarrow q_{b}, a\left(q_{b}\right) \rightarrow q_{f}\right\}$. Thus, $\mathcal{L}(\mathcal{A})=$ $\{a(b(\omega))\}$. Given the TRS $\mathcal{R}=\{a(x) \rightarrow c(d(x)), b(x) \rightarrow e(f(x))\}$, two critical pairs are computed: $a\left(q_{b}\right) \rightarrow_{\mathcal{A}}^{*} q_{f}, a\left(q_{b}\right) \rightarrow_{\mathcal{R}} c\left(d\left(q_{b}\right)\right)$ and $b\left(q_{\omega}\right) \rightarrow_{\mathcal{A}}^{*} b\left(q_{\omega}\right) \rightarrow_{\mathcal{R}} e\left(f\left(q_{\omega}\right)\right)$. Let $\gamma$ be the abstraction function such that $\gamma\left(a(x) \rightarrow c(d(x)),\left\{x \rightarrow q_{b}\right\}, q_{f}\right)(\epsilon)=q_{f}$, $\gamma\left(a(x) \rightarrow c(d(x)),\left\{x \rightarrow q_{b}\right\}, q_{f}\right)(1)=q_{f}, \gamma\left(b(x) \rightarrow e(f(x)),\left\{x \rightarrow q_{\omega}\right\}, q_{b}\right)(\epsilon)=q_{b}$ and $\gamma\left(b(x) \rightarrow e(f(x)),\left\{x \rightarrow q_{\omega}\right\}, q_{b}\right)(1)=q_{b}$. So, $\operatorname{Norm}_{\gamma}(a(x) \rightarrow c(d(x)),\{x \rightarrow$ $\left.\left.q_{b}\right\}, q_{f}\right)=\left\{d\left(q_{b}\right) \rightarrow q_{f}, c\left(q_{f}\right) \rightarrow q_{f}\right\}$ and $\operatorname{Norm}_{\gamma}\left(b(x) \rightarrow e(f(x)),\left\{x \rightarrow q_{\omega}\right\}, q_{b}\right)=$ $\left\{f\left(q_{\omega}\right) \rightarrow q_{b}, e\left(q_{b}\right) \rightarrow q_{b}\right\}$.

Now we formally define what a completion step is. Let $\mathcal{A}=\left\langle\mathcal{F}, \mathcal{Q}, \mathcal{Q}_{f}, \Delta\right\rangle$ be a tree automaton, $\gamma$ an abstraction function and $\mathcal{R}$ a left-linear TRS. We define a tree automaton $\mathcal{C}_{\gamma}^{\mathcal{R}}(\mathcal{A})=\left\langle\mathcal{F}, \mathcal{Q}^{\prime}, \mathcal{Q}_{f}^{\prime}, \Delta^{\prime}\right\rangle$ with:

- $\Delta^{\prime}=\Delta \cup \bigcup_{l \rightarrow r \in R, \sigma: \mathcal{X} \mapsto \mathcal{Q}, l \sigma \rightarrow{ }_{\mathcal{A}}^{*} q, r \sigma \nrightarrow{ }_{\mathcal{A}}^{*} q} \operatorname{Norm}_{\gamma}(l \rightarrow r, \sigma, q)$,

- $Q^{\prime}=\left\{q \mid c \rightarrow q \in \Delta^{\prime}\right\}$ and

- $Q_{f}^{\prime}=\mathcal{Q}_{f}$.

Example 2. Given $\mathcal{A}, \mathcal{R}$ and $\gamma$ of Example 1, performing one completion step on $\mathcal{A}$ gives the automaton $\mathcal{C}_{\gamma}^{\mathcal{R}}(\mathcal{A})$ such that $\mathcal{C}_{\gamma}^{\mathcal{R}}(\mathcal{A})=\left\langle\mathcal{F}, \mathcal{Q}, \mathcal{Q}_{f}, \Delta^{\prime}\right\rangle$ where $\Delta^{\prime}=$ $\Delta \cup N^{\prime r m} m_{\gamma}\left(a(x) \rightarrow c(d(x)),\left\{x \rightarrow q_{b}\right\}, q_{f}\right) \cup N \operatorname{Norm}_{\gamma}\left(b(x) \rightarrow e(f(x)),\left\{x \rightarrow q_{\omega}\right\}, q_{b}\right)=$ $\left\{\omega \rightarrow q_{\omega}, b\left(q_{\omega}\right) \rightarrow q_{b}, a\left(q_{b}\right) \rightarrow q_{f}, d\left(q_{b}\right) \rightarrow q_{f}, c\left(q_{f}\right) \rightarrow q_{f}, f\left(q_{\omega}\right) \rightarrow q_{b}, e\left(q_{b}\right) \rightarrow q_{b}\right\}$. Notice that $\mathcal{C}_{\gamma}^{\mathcal{R}}(\mathcal{A})$ is $\mathcal{R}$-close, and in fact an over-approximation of $\mathcal{R}^{*}(\mathcal{L}(\mathcal{A}))$ is computed. Indeed, the tree automaton $\mathcal{C}_{\gamma}^{\mathcal{R}}(\mathcal{A})$ recognises the term a $(e(e(f(\omega))))$ when

$$
\mathcal{R}^{*}(\mathcal{L}(\mathcal{A}))=\{a(b(\omega)), a(e(f(\omega))), c(d(b(\omega))), c(d(e(f(\omega))))\} .
$$

Proposition 3 (Theorem 1 in [17]) Let $\mathcal{A}$ be a tree automaton and $\mathcal{R}$ be a TRS such that $\mathcal{A}$ is deterministic or $\mathcal{R}$ is left-linear, and for every $l \rightarrow r \in \mathcal{R}, \mathcal{V}$ ar $(r) \subseteq$ $\mathcal{V} a r(l)$. For any abstraction function $\gamma$, one has:

$$
\mathcal{L}(\mathcal{A}) \cup \mathcal{R}(\mathcal{L}(\mathcal{A})) \subseteq \mathcal{C}_{\gamma}^{\mathcal{R}}(\mathcal{A})
$$


In addition, an abstraction function can be defined in such a way that only terms, actually reachable, will be computed. This class of abstraction functions is called $(\mathcal{A}, \mathcal{R})$-exact abstraction functions in [3].

Let $\mathcal{A}=\left\langle\mathcal{F}, \mathcal{Q}, \mathcal{Q}_{f}, \Delta\right\rangle$ be a tree automaton and $\mathcal{R}$ be a TRS. Let $\operatorname{Im}(\gamma)=\{q \mid$ $\forall l \rightarrow r \in \mathcal{R}, \forall p \in \mathcal{P o s}_{\mathcal{F}}(r)$ s.t. $\left.\gamma(l \rightarrow r, \sigma, q)(p)=q\right\}$. An abstraction function $\gamma$ is $(\mathcal{A}, \mathcal{R})-$ exact if $\gamma$ is injective and $\operatorname{Im}(\gamma) \cap \mathcal{Q}=\emptyset$.

By adapting the proof of Theorem 2 in [17] to the new class of abstractions, we show that with such abstraction functions, only reachable terms are computed.

Theorem 4 (Theorem 2 in [17]) Let $\mathcal{A}$ be a tree automaton and $\mathcal{R}$ be a TRS such that $\mathcal{A}$ is deterministic or $\mathcal{R}$ is right-linear. Let $\alpha$ be an $(\mathcal{A}, \mathcal{R})$-exact abstraction function. One has: $\mathcal{C}_{\alpha}^{\mathcal{R}}(\mathcal{A}) \subseteq \mathcal{R}^{*}(\mathcal{L}(\mathcal{A}))$.

We now give the general result in [17] saying that, if there exists a fix-point automaton, then its language contains all the terms actually reachable by rewriting, at least.

Theorem 5 (Theorem 1 in [17]) Let $\mathcal{A}, \mathcal{R}$ and $\gamma$ be respectively a tree automaton, a TRS. For any abstraction function, if there exists $N \in \mathbb{N}$ and $N \geq 0$ such that $\left(\mathcal{C}_{\gamma}^{\mathcal{R}}\right)^{(N)}(\mathcal{A})=\left(\mathcal{C}_{\gamma}^{\mathcal{R}}\right)^{(N+1)}(\mathcal{A})$, then $\mathcal{R}^{*}(\mathcal{L}(\mathcal{A})) \subseteq \mathcal{L}\left(\left(\mathcal{C}_{\gamma}^{\mathcal{R}}\right)^{(N)}(\mathcal{A})\right)$.

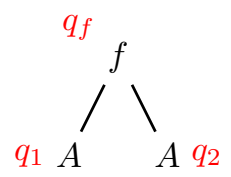

Figure 2. A run of $\mathcal{A}$ on $f(A, A)$.
The above method does not work for all TRSs. For instance, consider a constant $A$ and the tree automaton $\mathcal{A}=\left(\left\{q_{1}, q_{2}, q_{f}\right\},\left\{A \rightarrow q_{1}, A \rightarrow\right.\right.$ $\left.\left.q_{2}, f\left(q_{1}, q_{2}\right) \rightarrow q_{f}\right\},\left\{q_{f}\right\}\right)$ (Fig. 2) and the TRS $\mathcal{R}=\{f(x, x) \rightarrow g(x)\}$. There is no substitution $\sigma$ such that $l \sigma \rightarrow_{\mathcal{A}}^{*} q$, for a $q$ in $\left\{q_{1}, q_{2}, q_{f}\right\}$. Thus, following the procedure, there is no transition to add. But $f(A, A) \in \mathcal{L}(\mathcal{A})$. Thus $g(A) \in \mathcal{R}(\mathcal{L}(\mathcal{A}))$. Since $g(A) \notin \mathcal{L}(\mathcal{A})$, the procedure stops (in fact does not begin) before providing an over-approximation of $\mathcal{R}^{*}(\mathcal{L}(\mathcal{A}))$.

\section{Contributions}

This section extends an approximation-based technique introduced in [17] for leftlinear term-rewriting systems, to TRSs with non left-linear rules. If the termrewriting system is not left-linear, it is possible to apply Proposition 3 by determinizing the envolved automaton. However, since the determinisation is exponential and since it has to be done at each completion step (which doesn't preserve the determinism), the procedure is too expensive to be used in practice. In this framework, 
our contribution provides a well-suited (parametrised) model and underpinning theoretical tools that avoid these exponential steps when the term rewriting system is $h$-left-linear, with a small $h$. The small values of $h$ can be justified by the fact that most of TRS's modelling concrete verification problems are 2-left-linear.

Definition 6. Let $\mathcal{A}=\left(\mathcal{Q}, \Delta, \mathcal{Q}_{f}\right)$ be a finite bottom-up tree automaton. The automaton $\mathcal{A}^{(h)}=\left(\mathcal{Q}^{(h)}, \Delta^{(h)}, \mathcal{Q}_{f}^{(h)}\right)$, for $h \geq 1$, is defined by:

- $\mathcal{Q}^{(h)}=\{\{q\} \mid q \in \mathcal{Q}\} \cup\{Q \subseteq \mathcal{Q} \mid \mathcal{C} \operatorname{ard} Q \leq h\}$ (states of $\mathcal{Q}^{(h)}$ are denoted with an (h) exponent),

- $\mathcal{Q}_{f}^{(h)}=\left\{\{q\} \mid q \in \mathcal{Q}_{f}\right\}$,

- $\Delta^{(h)}=\left\{f\left(q_{1}^{(h)}, \ldots, q_{n}^{(h)}\right) \rightarrow q^{(h)} \mid \forall q \in q^{(h)}, \exists q_{1}, \ldots, q_{n} \in \mathcal{Q}, \forall 1 \leq i \leq\right.$ $n, q_{i} \in q_{i}^{(h)}$ and $\left.f\left(q_{1}, \ldots, q_{n}\right) \rightarrow q \in \Delta\right\}$.

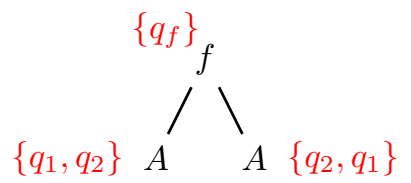

Figure 3. A run of $\mathcal{A}^{(2)}$ on $f(A, A)$.
To illustrate the definition above, let's consider the automaton $\mathcal{A}$ whose final state is $q_{f}$ and whose transitions are $A \rightarrow q_{1}, A \rightarrow q_{2}$ and $f\left(q_{1}, q_{2}\right) \rightarrow q_{f}$. The states of $\mathcal{A}^{(2)}$ are all pairs of states and singletons over $\left\{q_{1}, q_{2}, q_{f}\right\}$, and the transitions are $A \rightarrow\left\{q_{1}\right\}, A \rightarrow$ $\left\{q_{2}\right\}, A \rightarrow\left\{q_{1}, q_{2}\right\}, f\left(\left\{q_{1}\right\},\left\{q_{2}\right\}\right) \rightarrow$ $\left\{q_{f}\right\}, f\left(\left\{q_{1}, q_{i}\right\},\left\{q_{2}, q_{j}\right\}\right) \rightarrow\left\{q_{f}\right\}$ for all $i, j$ 's in $\{1,2, f\}$ (Fig. 3). When considering only the accessible states, among all the transitions above we just have transitions $f\left(\left\{q_{1}, q_{i}\right\},\left\{q_{2}, q_{j}\right\}\right) \rightarrow$ $\left\{q_{f}\right\}$ for all $i, j$ 's in $\{1,2\}$.

Proposition 7. One has $\mathcal{L}(\mathcal{A})=\mathcal{L}\left(\mathcal{A}^{(h)}\right)$.

Proof. By definition of $\mathcal{A}^{(h)}$, if $f\left(q_{1}, \ldots, q_{n}\right) \rightarrow q \in \Delta$, then $f\left(\left\{q_{1}\right\}, \ldots,\left\{q_{n}\right\}\right) \rightarrow$ $\{q\} \in \Delta^{(h)}$. Consequently, for every term $t$ such that $t \rightarrow_{\mathcal{A}}^{*} q$, one also has $t \rightarrow_{\mathcal{A}^{(h)}}^{*}$ $\{q\}$. Since for every $q_{f} \in \mathcal{Q}_{f},\left\{q_{f}\right\} \in \mathcal{Q}_{f}^{(h)}, \mathcal{L}(\mathcal{A}) \subseteq \mathcal{L}\left(\mathcal{A}^{(h)}\right)$.

It remains to prove that $\mathcal{L}\left(\mathcal{A}^{(h)}\right) \subseteq \mathcal{L}(\mathcal{A})$. We will prove by induction on $k$ that for every $k \geq 1$, for every term $t$, every state $q^{(h)}$ of $\mathcal{A}^{(h)}$, if $t \rightarrow{ }_{\mathcal{A}^{(h)}}^{k} q^{(h)}$, then for all $q \in q^{(h)}, t \rightarrow{ }_{\mathcal{A}}^{k} q$.

- If $t \rightarrow{ }_{A^{(h)}} q^{(h)}$, then, by definition of $\Delta^{(h)}, t$ is a constant and for all $q \in q^{(h)}$, there exists a transition $t \rightarrow q$ in $\mathcal{A}$.

- Assume now that the claim is true for a fixed positive integer $k$. Let $t$ be a term and $q^{(h)} \in \mathcal{A}^{(h)}$ such that $t \rightarrow_{\mathcal{A}^{(h)}}^{k+1} q^{(h)}$. Consequently, there exists 
$f \in \mathcal{F}_{n}$ such that $t \rightarrow_{\mathcal{A}^{(h)}}^{k} f\left(q_{1}^{(h)}, \ldots \ldots, q_{n}^{(h)}\right) \rightarrow_{\mathcal{A}^{(h)}} q^{(h)}$. It follows that $t=f\left(t_{1}, \ldots, t_{k}\right)$ and for all $1 \leq i \leq k, t_{i} \rightarrow_{\mathcal{A}^{(h)}}^{k} q_{i}^{(h)}$. Using the induction hypothesis, $t_{i} \rightarrow_{\mathcal{A}}^{k} q_{i}$, for all $q_{i} \in q_{i}^{(h)}$. Consequently, for all $q \in q^{(h)}$, $f\left(q_{1}, \ldots, q_{n}\right) \rightarrow q \in \Delta$, proving the induction.

So, $\mathcal{L}\left(\mathcal{A}^{(h)}\right) \subseteq \mathcal{L}(\mathcal{A})$.

Lemma 8. Let $k \geq 1$. If $C\left[q_{1}, \ldots, q_{n}\right] \rightarrow_{\mathcal{A}}^{k} q$ and if $q_{1}^{(h)}, \ldots q_{n}^{(h)}$ are states of $\mathcal{A}^{(h)}$ satisfying $q_{i} \in q_{i}^{(h)}$ for all $1 \leq i \leq n$, then $C\left[q_{1}^{(h)}, \ldots, q_{n}^{(h)}\right] \rightarrow_{\mathcal{A}^{(h)}}^{k}\{q\}$.

Proof. We prove by induction on $k$ that for every $k \geq 1$, if $C\left[q_{1}, \ldots, q_{n}\right] \rightarrow_{\mathcal{A}}^{k} q$ and if $q_{1}^{(h)}, \ldots q_{n}^{(h)}$ are states of $\mathcal{A}^{(h)}$ satisfying $q_{i} \in q_{i}^{(h)}$ for all $1 \leq i \leq n$, then $C\left[q_{1}^{(h)}, \ldots, q_{n}^{(h)}\right] \rightarrow_{\mathcal{A}^{(h)}}^{k}\{q\}$.

- If $k=1$, then $C\left[q_{1}, \ldots, q_{n}\right] \rightarrow q$ is a transition of $\mathcal{A}$. Therefore, by definition of $\Delta^{(h)}, C\left[q_{1}^{(h)}, \ldots, q_{n}^{(h)}\right] \rightarrow\{q\}$ is a transition of $\mathcal{A}^{(h)}$.

- Assume now that the proposition is true for all $j \leq k$ and that $C\left[q_{1}, \ldots, q_{n}\right] \rightarrow_{\mathcal{A}}^{k+1} q$. There exist $q_{1}^{\prime}, \ldots, q_{\ell}^{\prime}$ states of $\mathcal{A}$ and $f \in \mathcal{F}_{\ell}$ such that $C\left[q_{1}, \ldots, q_{n}\right] \rightarrow_{\mathcal{A}}^{k} f\left(q_{1}^{\prime}, \ldots, q_{\ell}^{\prime}\right) \rightarrow_{\mathcal{A}} q$. Consequently, $C\left[q_{1}, \ldots, q_{n}\right]$ is of the form $C\left[q_{1}, \ldots, q_{n}\right]=f\left(t_{1}, \ldots, t_{\ell}\right)$ where the $t_{i}$ 's are terms over $\mathcal{F} \cup\left\{q_{1}, \ldots, q_{n}\right\}$. Moreover, for all $i$, there exists $k_{i} \leq k$ such that $t_{i} \rightarrow_{\mathcal{A}}^{k_{i}}\left\{q_{i}^{\prime}\right\}$ and $\sum_{i} k_{i}=k$. Therefore, by induction hypothesis, $t_{i}^{(h)} \rightarrow_{{ }_{\mathcal{A}}^{(h)}}^{k_{i}}\left\{q_{i}^{\prime}\right\}$ where $t_{i}^{(h)}$ is the term obtained from $t_{i}$ by substituting $q_{i}$ by $q_{i}^{(h)}$. Now, since $f\left(q_{1}^{\prime}, \ldots, q_{\ell}^{\prime}\right) \rightarrow q$ is a transition of $\mathcal{A}, f\left(\left\{q_{1}^{\prime}\right\}, \ldots,\left\{q_{\ell}^{\prime}\right\}\right) \rightarrow\{q\}$ is a transition of $\mathcal{A}^{(h)}$.

It follows that $C\left[q_{1}^{(h)}, \ldots, q_{n}^{(h)}\right] \rightarrow_{\mathcal{A}^{(h)}}^{k+1}\{q\}$, proving the lemma.

Lemma 9. If there are $q_{1}, q_{2}, \ldots, q_{j}$ states of $\mathcal{A}$, with $j \leq h$ such that $t \rightarrow_{\mathcal{A}}^{*} q_{i}$ for every $1 \leq i \leq j$, then $t \rightarrow_{\mathcal{A}^{(h)}}^{*}\left\{q_{i} \mid 1 \leq i \leq j\right\}$.

Proof. If $t \rightarrow{ }_{\mathcal{A}}^{*} q_{i}$ for every $1 \leq i \leq j$, then there exist functions $\pi_{i}$ 's from positions of $t$ into $\mathcal{Q}$ such that $\pi_{i}(\varepsilon)=q_{i}$ and for every position $p$ of $t$, if $\left.t\right|_{p} \in \mathcal{F}_{n}$, then $t(p)\left(\pi_{i}(p .1), \ldots, \pi_{i}(p . n)\right) \rightarrow \pi_{i}(p)$ is a transition of $\mathcal{A}$.

Therefore, by definition of $\Delta^{(h)}, t(p)\left(\left\{\pi_{i}(p .1) \mid 1 \leq i \leq j\right\}, \ldots,\left\{\pi_{i}(p . n) \mid 1 \leq\right.\right.$ $i \leq j\}) \rightarrow\left\{\pi_{i}(p) \mid 1 \leq i \leq j\right\}$ is in $\Delta^{(h)}$. It follows that $t \rightarrow_{\mathcal{A}^{(h)}}^{*}\left\{q_{i} \mid 1 \leq i \leq j\right\}$.

Proposition 10. If each variable occurs at most $h$ times in left-hand sides of rules of $\mathcal{R}$, then $\mathcal{R}(\mathcal{L}(\mathcal{A})) \cup \mathcal{L}(\mathcal{A}) \subseteq \mathcal{L}\left(\mathcal{C}_{\gamma}^{\mathcal{R}}\left(\mathcal{A}^{(h)}\right)\right)$.

Proof. Since $\mathcal{L}(\mathcal{A})=\mathcal{L}\left(\mathcal{A}^{(h)}\right)$ and since $\mathcal{L}\left(\mathcal{A}^{(h)}\right) \subseteq \mathcal{L}\left(\mathcal{C}_{\gamma}^{\mathcal{R}}\left(\mathcal{A}^{(h)}\right)\right), \mathcal{L}(\mathcal{A}) \subseteq$ $\mathcal{L}\left(\mathcal{C}_{\gamma}^{\mathcal{R}}\left(\mathcal{A}^{(h)}\right)\right)$. It remains to show that $\mathcal{R}(\mathcal{L}(\mathcal{A})) \subseteq \mathcal{L}\left(\mathcal{C}_{\gamma}^{\mathcal{R}}\left(\mathcal{A}^{(h)}\right)\right)$. 
Let $t \in \mathcal{R}(\mathcal{L}(\mathcal{A}))$. By definition there exists a rule $l \rightarrow r \in \mathcal{R}$, a position $p$ of $t$ and a substitution $\mu$ from $\mathcal{X}$ into $\mathcal{T}(\mathcal{F})$ such that

$$
t=t[r \mu]_{p} \quad \text { and } \quad t[l \mu]_{p} \in \mathcal{L}(\mathcal{A})
$$

It follows there exist states $q, q_{f}$ of $\mathcal{A}$ such that $q_{f}$ is final, and

$$
l \mu \rightarrow{ }_{\mathcal{A}}^{*} q \quad \text { and } \quad t[q]_{p} \rightarrow{ }_{\mathcal{A}}^{*} q_{f} .
$$

Consequently, by Definition 6, one has

$$
l \mu \rightarrow_{\mathcal{A}^{(h)}}^{*}\{q\} \quad \text { and } \quad t[\{q\}]_{p} \rightarrow_{\mathcal{A}^{(h)}}^{*}\left\{q_{f}\right\} .
$$

If $r \mu \rightarrow_{\mathcal{A}^{(h)}}^{*}\{q\}$, then (3) implies that $t[r \mu]_{p} \rightarrow_{\mathcal{A}^{(h)}}^{*}\left\{q_{f}\right\}$. In this case, since $t=$ $t[r \mu]_{p}$ and since $\left\{q_{f}\right\}$ is by construction a final state of $\mathcal{A}^{(h)}, t$ is in $\mathcal{L}\left(\mathcal{A}^{(h)}\right)$, which is a subset of $\mathcal{L}\left(\mathcal{C}_{\gamma}\left(\mathcal{A}^{(h)}\right)\right)$.

Now we may assume that $r \mu \rightarrow_{\mathcal{A}^{(h)}}^{*}\{q\}$. Let $P_{l}$ be the set of variable positions of $l$; i.e. $\left.P_{l}=\{p \mid l(p) \in \mathcal{X})\right\}$. Set $P_{l}=\left\{p_{1}, \ldots, p_{\ell}\right\}$. Since $l \mu \rightarrow_{\mathcal{A}}^{*} q$, by (2) there exist states $q_{1}, \ldots, q_{\ell}$ of $\mathcal{A}$ such that

$$
\mu\left(l\left(p_{i}\right)\right) \rightarrow_{\mathcal{A}}^{*} q_{i} \quad \text { and } \quad l\left[q_{1}\right]_{p_{1}} \ldots\left[q_{\ell}\right]_{p_{\ell}} \rightarrow{ }_{\mathcal{A}}^{*} q .
$$

We define the substitution $\sigma$ from variables occurring in $l$ into $2^{\mathcal{Q}}$ by: $\sigma\left(x_{i}\right)=\left\{q_{i} \mid\right.$ $\left.l\left(p_{i}\right)=x_{i}\right\}$. Since $l$ is h-left-linear, for each $x_{i}, \sigma\left(x_{i}\right)$ contains at most $\mathrm{h}$ states. We claim that $l \sigma \rightarrow_{\mathcal{A}^{(h)}}^{*} q$. Indeed by (4) and by Lemma 9 for each $x_{i}$ occurring in $l, \mu\left(x_{i}\right) \rightarrow_{\mathcal{A}^{(h)}}^{*} \sigma\left(x_{i}\right)$. It follows that $l \mu \rightarrow_{\mathcal{A}^{(h)}}^{*} l \sigma$. By (4) and using Lemma 8, $l \sigma \rightarrow_{\mathcal{A}^{(}(h)}^{*}\{q\}$, proving the claim. By construction of $\mathcal{C}_{\gamma}^{\mathcal{R}}\left(\mathcal{A}^{(h)}\right), r \sigma \rightarrow_{\mathcal{C}_{\gamma}^{\mathcal{R}}\left(\mathcal{A}^{(h)}\right)}^{*}\{q\}$. Moreover, by definition of $\sigma, r \mu \rightarrow_{\mathcal{A}^{(}(h)}^{*} r \sigma$. It follows that

$$
t=t[r \mu]_{p} \rightarrow_{\mathcal{A}^{(h)}}^{*} t[r \sigma]_{p} \rightarrow_{\mathcal{C}_{\gamma}\left(\mathcal{A}^{(h)}\right)}^{*} t[\{q\}]_{p} \rightarrow_{\mathcal{A}^{(h)}}^{*}\left\{q_{f}\right\},
$$

which completes the proof.

Proposition 11. If $\mathcal{R}$ is right-linear and if $\alpha$ is $(\mathcal{A}, \mathcal{R})$-exact, then $\mathcal{L}\left(\mathcal{C}_{\alpha}\left(\mathcal{A}^{(h)}\right)\right) \subseteq$ $\mathcal{R}^{*}(\mathcal{L}(\mathcal{A}))$.

Proof. This is a direct consequence of Theorem 4 and Proposition 7.

Notice that if $\mathcal{A}$ is a non-deterministic $n$-state automaton, any determinitic automaton recognising $\mathcal{L}(\mathcal{A})$ may have $O\left(2^{n}\right)$ states. Furthermore, $\mathcal{A}^{(h)}$ has $O\left(n^{h}\right)$ states. For 2-left-linear term-rewriting systems, $\mathcal{A}^{(h)}$ has significantly less states that a deterministic automaton accepting $\mathcal{L}(\mathcal{A})$.

\section{Example and Application Domains}

\subsection{Example}

We have tested our approach on the following family of examples. We first consider a family of tree automata $\left(\mathcal{A}_{n}\right)$ defined as follows: the set of states of $\mathcal{A}_{n}$ 
is $\left\{q_{1}, \ldots, q_{2 n+2}, q_{f}\right\}$, the set of final state is $\left\{q_{f}\right\}$, and the set of transitions is $\left\{\omega \rightarrow q_{1}, \omega \rightarrow q_{2}, a\left(q_{1}\right) \rightarrow q_{1}, a\left(q_{2}\right) \rightarrow q_{2}, b\left(q_{1}\right) \rightarrow q_{1}, b\left(q_{2}\right) \rightarrow q_{2}, a\left(q_{1}\right) \rightarrow\right.$ $\left.q_{3}, a\left(q_{2}\right) \rightarrow q_{4}, a\left(q_{i}\right) \rightarrow q_{i+2}, b\left(q_{i}\right) \rightarrow q_{i+2}, f\left(q_{2 n+1}, q_{2 n+2}\right) \rightarrow q_{f}\right\}$, for $i \geq 3$. The automaton $\mathcal{A}_{n}$ accepts the set of terms of the form $f\left(t_{1}, t_{2}\right)$ where $t_{1}$ and $t_{2}$ are terms over $\{a, b, \omega\}$ such that $\left.t_{1}\right|_{1^{n-1}}$ and $\left.t_{2}\right|_{1^{n-1}}$ exist and are in $\{a\} .\{a, b\}^{*}$. Roughly speaking, when using word automata, $a(b(\omega))$ denotes $a b$, and each pair $\left(t_{1}, t_{2}\right)$ can be viewed as words of $\mathcal{L}=\{a, b\}^{n-1} \cdot\{a\} .\{a, b\}^{*}$ satisfying the condition above. We second consider the term rewriting system $\mathcal{R}$ containing the single rule $f(x, x) \rightarrow x$, and we want to prove that $b^{n-1} a(\omega) \in \mathcal{R}^{*}\left(\mathcal{L}\left(\mathcal{A}_{n}\right)\right)$. Using finitely many times Theorem 4 directly on $\mathcal{A}_{n}$ may not prove the results. However, to prove the results, one can determinise $\mathcal{A}_{n}$ before using Theorem 4. But, the minimal automaton of $\mathcal{L}\left(\mathcal{A}_{n}\right)$ has $2^{n}$ states at least [21], [Exercise 3.20, p. 73]. Then, the completion should be applied to this automaton. Consequently, this automatic proof requires an exponential time step. Using our approach, one can compute $\mathcal{A}^{(h)}$ and apply Proposition 11, that provides the proof requiring a polynomial time step.

\subsection{Left-linearity and Security Issues}

\subsubsection{Security Protocol Analysis}

The TRSs used in the security protocol verification context are often non left-linear. Indeed, there is a lot of protocols that cannot be modeled by left-linear TRSs. Unfortunately, to be sound, the approximation-based analysis described in [18] requires the use of left-linear TRSs. Nevertheless, this method can still be applied to some non left-linear TRSs, which satisfy some weaker conditions. In [17] the authors propose new linearity conditions. However, these new conditions are not well-adapted to be automatically checked.

In our previous work [5] we explain how to define a criterion on $\mathcal{R}$ and $\mathcal{A}$ to make the procedure automatically work for industrial protocols analysis. This criterion ensures the soundness of the method described in $[18,17]$. However, to handle protocols the approach in [5] is based on a kind of constant typing. In [6] we go further and propose a procedure supporting a fully automatic analysis and handling - without typing - algebraic properties like XOR.

Let us first remark that the criterion defined in [17] does not allow managing the XOR non left-linear rule. Second, in [5] we have restricted XOR operations to typed terms to deal with the XOR non left-linear rule. However, some protocols are known to be flawed by type confusing attacks $[14,10,11]$. Notice that our approach in [6] can be applied to any kinds of TRSs. Moreover, it can cope with exponentiation algebraic properties and this way analyse Diffie-Hellman based protocols.

\subsubsection{Backward Analysis of Java Bytecode}

A recent work [4], dedicated to the static analysis of Java bytecode programs using term-rewriting systems, provides an automatic procedure to translate a Java byte- 
code into a term rewriting system modeling the code execution on the Java Virtual Machine. In this context, generated TRSs are left-linear but non right-linear. For example the rewriting rule:

$$
\begin{aligned}
& \left.x \text { InvokeSpecialCC (pprotected, val } l_{\text {true }}, c c, c a, c a m, i c\right) \rightarrow \\
& x \text { InvokeSpecialCC }\left(\text { subclass }(i c, c c), v_{\text {true }}, c c, c a, c a m, i c\right)
\end{aligned}
$$

where $c a, c a m, i c$ and $c c$ are variables, is a 2-right-linear rule. This rule is a part of a Java bytecode instruction translation, corresponding to the invocation of the method. In order to compute approximation refinements as in [3] or to manage backward analyses that are - in general and in practice - more efficient that forward analyses - term rewriting systems have to be turned left-right, i.e. left- and righthand sides of rules have to be permuted. By this permutation 2-right-linear TRSs become 2-left-linear ones.

\section{Conclusion}

Regular approximation techniques have been successfully used in the context of security protocol analysis. In order to apply them to other applications, this paper proposed an extension of the completion procedure for handling non left-linear rules. Our contributions allow analysing some reachability problems using polynomial steps computing $\mathcal{A}^{(h)}$, rather than automata determinisation steps that are exponential, even in practical cases. Notice that the approach presented only for quadratic rules can be extended to more complex TRSs. We intend to optimise this technique: polynomial is better than exponential but may also lead to huge automata in few steps. We have been implementing the techniques in an efficient rewriting tool like $\mathrm{TOM}^{\mathrm{a}}$ in order to investigate complex systems backward analyses.

\section{Bibliography}

[1] P. A. Abdulla, B. Jonsson, P. Mahata, and J. d'Orso. Regular tree model checking. In E. Brinksma and K. G. Larsen, editors, Computer Aided Verification, volume 2404 of Lecture Notes in Computer Science, pages 555-568. Springer-Verlag, July 27-31 2002.

[2] F. Baader and T. Nipkow. Term Rewriting and All That. Cambridge University Press, 1998.

[3] Y. Boichut, R. Courbis, P.-C. Héam, and O. Kouchnarenko. Finer is better: Abstraction refinement for rewriting approximations. In RTA'08, 19th int. conf. on Rewriting Techniques and Applications, LNCS, 2008.

[4] Y. Boichut, Th. Genet, Th. P. Jensen, and L. Le Roux. Rewriting approximations for fast prototyping of static analyzers. In Franz Baader, editor, Term Rewriting and Applications, 18th International Conference, RTA 200\%, Paris, France, June 26-28, 2007, Proceedings, volume 4533 of LNCS, pages 48-62. Springer, 2007.

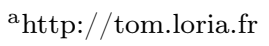


[5] Y. Boichut, P.-C. Héam, and O. Kouchnarenko. Automatic Verification of Security Protocols Using Approximations. Technical Report RR-5727, INRIA, 2005.

[6] Y. Boichut, P.-C. Héam, and O. Kouchnarenko. Handling algebraic properties in automatic analysis of security protocols. In Kamel Barkaoui, Ana Cavalcanti, and Antonio Cerone, editors, ICTAC'06, volume 4281 of $L N C S$, pages 153-167. Springer, 2006.

[7] Y. Boichut and P.-C. Héam. A theorerical limit for safety verification techniques with regular fix-point computations. Information Processing Letters, 108(1):1-2, September 2008 .

[8] A. Bouajjani, P. Habermehl, A. Rogalewicz, and T. Vojnar. Abstract regular tree model checking. In Infinity'05, volume 149 of Electronic Notes in Theoretical Computer Science, pages 37-48, 2006.

[9] A. Bouajjani and T. Touili. Extrapolating tree transformations. In Ed Brinksma and Kim Guldstrand Larsen, editors, Computer Aided Verification, volume 2404 of Lecture Notes in Computer Science, pages 539-554. Springer-Verlag, July 27-31 2002.

[10] L. Bozga, Y. Lakhnech, and M. Perin. Pattern-based abstraction for verifying secrecy in protocols. In 9th International Conference on Tools and Algorithms for the Construction and Analysis of Systems, TACAS 2003, Proceedings, volume 2619 of LNCS. Springer-Verlag, 2003.

[11] I. Cibrario, L. Durante, R. Sisto, and A. Valenzano. Automatic detection of attacks on cryptographic protocols: A case study. In Christopher Kruegel Klaus Julisch, editor, Intrusion and Malware Detection and Vulnerability Assessment: Second International Conference, volume 3548 of LNCS, Vienna, 2005.

[12] H. Comon, M. Dauchet, R. Gilleron, F. Jacquemard, D. Lugiez, S. Tison, and M. Tommasi. Tree Automata Techniques and Applications, 2002.

[13] J.-L. Coquidé, M. Dauchet, R. Gilleron, and Vágvölgyi S. Bottom-up tree pushdown automata and rewrite systems. In Ronald V. Book, editor, Rewriting Techniques and Applications, 4th International Conference, RTA-91, LNCS 488, pages 287-298, Como, Italy, April 10-12, 1991. Springer-Verlag.

[14] V. Cortier, S. Delaune, and P. Lafourcade. A survey of algebraic properties used in cryptographic protocols. Journal of Computer Security, 14:1-43, 2006.

[15] M. Dauchet and S. Tison. The theory of ground rewrite systems is decidable. In LICS: IEEE Symposium on Logic in Computer Science, 1990.

[16] N. Dershowitz and J.-P. Jouannaud. Handbook of Theoretical Computer Science, volume B, chapter 6: Rewrite Systems, pages 244-320. Elsevier Science Publishers B. V, 1990.

[17] G. Feuillade, Th. Genet, and V. VietTriemTong. Reachability analysis over term rewriting systems. Journal of Automated Reasonning, 33 (3-4), 2004.

[18] Th. Genet and F. Klay. Rewriting for Cryptographic Protocol Verification. In proceedings of CADE, volume 1831 of $L N C S$, pages 271-290. Springer-Verlag, 2000.

[19] R. Gilleron and S. Tison. Regular tree languages and rewrite systems. Fundamenta Informatica, 24(1/2):157-174, 1995.

[20] P. Habermehl, R. Iosif, A. Rogalewicz, and T. Vojnar. Abstract regular tree model checking of complex dynamic data structures. In $S A S^{\prime} 06$, 13th International Static Analysis Symposium, volume 4134 of LNCS, pages 52-70, 2006.

[21] J. Hopcroft and J. D. Ullman. Introduction to Automata Theory, Languages, and Computation. Addison-Wesley, 1979.

[22] F. Jacquemard. Decidable approximations of term rewriting systems. In proceedings of RTA, volume 1103, pages 362-376. Springer Verlag, 1996.

[23] T. Nagaya and Y. Toyama. Decidability for left-linear growing term rewriting sys- 
tems. In RTA '99: Proceedings of the 10th International Conference on Rewriting Techniques and Applications, pages 256-270, London, UK, 1999. Springer-Verlag.

[24] H. Ohsaki and T. Takai. ACTAS: A system design for associative and commutative tree automata theory. Electr. Notes Theor. Comput. Sci, 124(1):97-111, 2005.

[25] P. Réty and J. Vuotto. Regular sets of descendants by leftmost strategy. Electr. Notes Theor. Comput. Sci, 70(6), 2002.

[26] K. Salomaa. Deterministic tree pushdown automata and monadic tree rewriting systems. JCSS: Journal of Computer and System Sciences, 37, 1988. 